\title{
Overview of Experimental Studies on Strength Problem of Rubber
}

\section{Material}

\author{
Xuanli Zhang ${ }^{1, a^{*}}$, Guoying Zhao ${ }^{2, b}$ \\ ${ }^{1}$ College of Electromechanical Engineering, Qingdao University of Science and Technology, \\ Mechanics teaching and research section, Qingdao, 266061, China \\ ${ }^{2}$ College of Electromechanical Engineering, Qingdao University of Science and Technology, \\ Qingdao, 266061, China \\ azx16503@126.com, ${ }^{\mathrm{b}}$ zhaogy0321@126.com
}

\begin{abstract}
Keywords: rubber material, experimental study, fatigue problem, constitutive model, multiaxial load.

Abstract: The mechanical properties of rubber materials are complex, and the strength problem is getting more and more attention. This paper summarizes the research status of mechanics experiment of rubber materials under dynamic and static load, commented recent years domestic and foreign the progress on the field of rubber uniaxial and multiaxial fatigue. Introduced strength problem research under quasi static load of commonly used constitutive model, selection of constitutive model and determination of parameters status, pointed out the exist problems in experimental study of rubber materials strength.
\end{abstract}

\section{Introduction}

Rubber is a kind of important polymer material, which has the irreplaceable properties of other materials. Rubber material can withstand very large strain but does not produce permanent deformation or damage and has advantage that common engineering materials can not match, which makes it has very good application in many occasions. However, the mechanics behavior of rubber material complex, with a high degree of material nonlinearity and geometric nonlinearity, produce large defor- mation under the action of external force, which makes difficult of rubber materials properties and mechanical state has an accurate description. With the development and promotion of rubber materials, especially applied in high-tech industries, rubber material strength problem experimental research and mechanical state for precise description become more necessary and prominent.

\section{Rubber material fatigue problem}

\section{Study on fatigue under uniaxial loading}

Mechanical fatigue can be expressed as materials under dynamic load and deforma- tion, the slow growth of crack lead to gradual decrease of physical and mechanical properties. The fatigue test data of rubber material is basis of evaluating fatigue life of rubber material.

\section{Fatigue study based on strain amplitude, load frequency and minimum strain}

Early rubber fatigue study mainly used strain amplitude and minimum strain to describe failure load cycle number of rubber material. The influence of minimum strain on fatigue life of rubber was 
studied by Cadwell et $\mathrm{al}^{[1]}$. While rubber materials in uniaxial tensile and shear fatigue life has been studied by Cadwell, but the design of test specimens can not accurately achieve simple shear and pure tensile strain state, so the scope of application of experimental results exist certain limitations. Fielding ${ }^{[2]}$ proposed fatigue damage parameters of axial strain, and studied the effect of mini- mum strain on fatigue life of two new synthetic rubber, the results show that appropri- ately increased the minimum of loading strain can significant improve fatigue life.

In general, increase of the periodic load amplitude can lead to decrease fatigue life of rubber. Beatty ${ }^{[3]}$ study fatigue life of natural rubber found that rubber fatigue property improved when the periodic minimum strain amplitude is increased, indicat- ing that the minimum strain exerted on the test object is the main factors that affect fatigue life. Lee et al. ${ }^{[4]}$ carried out a lot of experiments study for tire fatigue property, and believed that the nonlinearity of rubber material is important factor caused the minimum strain influence life. Abraham ${ }^{[5]}$ through the filling of ethylene propylene rubber and styrene butadiene rubber and non filled EPR and SBR under single axial load characteristics, analyzed the minimum stress and stress amplitude on fatigue life influence of non-strain crystalline rubber. Mars ${ }^{[6]}$ used uniaxial tension specimen stud- ied crack nucleation life of rubber, indicate the effect of minimum strain on crack nucleation life is relatively large.

The effect of loading frequency depends on the type of polymer. For the strain crystalline rubber, loading frequency during $10^{-3}-5 \mathrm{~Hz}$ fatigue life was not changed. For amorphous rubber, the effect of loading frequency on fatigue life is remarkable ${ }^{[7]}$. When load frequency is too high, it will produce the heating phenomenon, resulted in temperature rise of rubber inside, thereby affecting fatigue resistance of rubber materials. Liu Yuyan ${ }^{[8]}$ used fatigue testing system by their own established, with unidirectional polyester cord reinforced rubber composite as the object, studied the factors affecting fatigue properties of rubber composites under cyclic loading. Obtain- ed stress amplitude and load frequency have a big influence on fatigue properties of rubber composites, and the average stress influence is small.

\section{Fatigue study based on the strain energy density evaluation parameters}

With the development of fracture mechanics, strain energy density is gradually introduced into the field of rubber fatigue life. Lee $\mathrm{M}$ P, Hocine $\mathrm{N}^{[9,10]}$ pointed out under the specific condition, energy release rate of rubber is proportional to the product of strain energy density and fracture size. They think that strain energy density is a measure of the intrinsic energy release rate in the specific crack size. MARS ${ }^{[11]}$ based on different engineering strain peak rubber stress-strain curves of established the peak power function relationship between strain energy density peak and engineering strain, by the engineering strain peak to obtain strain energy density peak. Roberts ${ }^{[12]}$ analyzed the relationship between energy release rate and lifetime by uniaxial experiments, compared with strain energy density, and some guidance sugge- stions for later stage research are given. Ro $\mathrm{H} \mathrm{S}^{[13]}$ reanalyzed the test data of Roberts, and found that strain energy density of high cycle fatigue range is a good parameter for rubber fatigue life evaluation. Yamashita ${ }^{[14]}$ by using strain energy density as fatigue damage parameters studied fatigue life of the shock absorber. Mars ${ }^{[6]}$ summa- rized the relationship between crack propagation life and strain energy function under uniaxial load. Abraham et al. ${ }^{[5]}$ based on the effect of minimum stress and stress amplitude on fatigue life of non strain crystalline rubber, proposed strain energy density can be used as the equivalent parameters of fatigue life. Zine et al. ${ }^{[15,16]}$ according to the proposed method of Mars, crack energy density and strain energy density are compared as damage parameters accuracy. The results show that cracking energy density is more suitable as damage 
parameters for the uniaxial tension and shear fatigue, but pointed out more tests should be carried out to verify accuracy of the conclusion.

\section{Current status of uniaxial load fatigue}

Roberts $\mathrm{J} \mathrm{B}$ et $\mathrm{al}^{\left[{ }^{[12]}\right.}$ through uniaxial tensile experimental, found that maximum princi- pal stress can be as an evaluation parameter for predict fatigue crack initiation life. Abraham ${ }^{[5]}$ found that maximum stress was the key to fatigue life during studied strain non crystalline rubber fatigue, but could not completely characterize fatigue life. Kim et $\mathrm{al}^{[17]}$ carry out uniaxial fatigue test of the dumbbell type rubber components, use maximum Lagrange strain fitted the data of fatigue life. They used finite element method, obtained fatigue life and maximum Green-Lagrange strain predict formula by using the dumbbell rubber column test. The fatigue life prediction of natural rubber components was studied by Woo et $\mathrm{al}^{[18]}$, and its research ideas were similar to that of Kim et al. Zhang Wei et $\mathrm{al}^{[19]}$ by SHPB experimental system of three different proportions of rubber for kinetic experiments study and analysis of rubber in different strain rate loading stress-strain characteristics, and based on experimental stress-strain curve, analysis dynamic fatigue property and relevant laws of different ratio of vulcanized rubber. Yin Suxian ${ }^{[20]}$ combination of use experimental and finite element analysis method, through uniaxial tension and planar tensile properties test of rubber materials, investigated the influence of radial pre compression on fatigue life of the rubber bushing. Xiao Suhua ${ }^{[21]}$ for fatigue life prediction of rubber isolator, based on fatigue life test data of the dumbbell rubber material, designed a dumbbell type rubber specimen carry out tensile fatigue test. Wang Xiaoli et al. ${ }^{[22]}$ carried out uniaxial tensile fatigue test of the specimens made of rubber, based on the experimental data of dumbbell specimen established rubber single shaft fatigue life prediction model. Obtained maximum principal strain peak, eight surface bulk shear strain and strain energy density peak as life prediction model of damage parameter can be well to power law to establish the relationship between damage parameter and fatigue life.

Because of the complex mechanical properties of rubber materials, the influence factors of its fatigue are numerous. Through investigation and experimental study of these factors, it can help to better understand the nature of rubber.

\section{Current status of multiaxial fatigue}

Only to the beginning of this century, more and more scholars under multiaxial cyclic loading develop rubber materials fatigue research, from both theoretical and experim- ental, rubber material multiaxial fatigue studies are in the initial stage.

Mars and Fatemi ${ }^{[23]}$ are the pioneers in the field of rubber multiaxial fatigue, and carried out a series of fatigue tests of filled type of natural vulcanized rubber under proportional and non proportional loading path. The results show that rubber cracks are nucleation and expansion of a certain plane under multiaxial fatigue. Saintier et al. ${ }^{[24]}$ studied failure plane is determined by the maximum principal stress direction, material plane and main stress surface can rotate under the large deformation. $\mathrm{Li}$ and Steif ${ }^{[25]}$ studied on mechanical behaviors of rubber material under proportional and 90 degrees of non proportional loading, results show that rubber material under cyclic loading stress response exhibits nonlinear properties, and main subject on the strain amplitude. Mars and Fatemi ${ }^{[26]}$ found in study of filled vulcanized natural rubber, cyclic stress-strain response existed obviously soften, and softening depends on the maximum strain, the bigger maximum strain and the bigger of stability response softening. Saintier et al. ${ }^{[24,27]}$ based on the theory of configuration stress tensor, established two fatigue evaluation criteria for dumbbell rubber columns used in evaluation of non proportional and multiaxial load cases. Andriyana. $\mathrm{A}^{[28,29]}$ from 
the point of engineering application reviewed configuration stress application in the study of rubber fatigue, based on configuration stress tensor method comprehensively considered the whole bearing and deformation process of rubber, can better predict the trend of rubber fatigue. The fatigue life data of MARS were analyzed by using this method, based on configuration stress tensor fatigue research method is suitable for multiaxial fatigue prediction of rubber materials was further considered.

$\mathrm{Yu}$ Weiwei ${ }^{[30]}$ found in study multiaxial cyclic characteristics of nitrile rubber, corresponding to the same equivalent strain, rubber under non proportional loading paths existed cyclic additional softening phenomenon. Ding Zhiping et al. ${ }^{[31]}$ through rubber pure shear specimen fatigue crack propagation tests, take tear energy a range as damage parameter, established under complex stress state of rubber fatigue crack propagation life prediction model. $\mathrm{Li}$ et $\mathrm{al}^{[32]}$ used maximum full principal strain parameters to evaluate fatigue life of rubber suspension, with logarithmic fatigue life formula for predicting fatigue life of rubber suspension and predict different direction deviation of fatigue life. Wang Yanping ${ }^{[33]}$ for two formulations filler type natural rubber carried out based on crack initiation of macro mechanical properties and based on crack growth microstructure correlation research, separately application strain energy density and tear energy to evaluate multiaxial fatigue life of rubber, and advantages and disadvantages of the two are compared. Wang Wentao, Shangguan Wenbin ${ }^{[34,35]}$ used measurement method studied calculation precision of rubber isolator finite element analysis method, proposed tensor form of rubber fatigue life prediction formula, and through experiment to test its accuracy.

Domestic and foreign scientists have made some achievements in the field of rubber multiaxial fatigue, due to large deformation characteristic of rubber material, the effective theory of rubber material has not been formed. Established unified viscoelastic constitutive model, for its fatigue properties effective predicting is the future further research direction.

\section{Research status of strength under quasi static load}

The experimental studies of strength under quasi static load of rubber materials are mainly concentrated in constitutive relation is presented, the choice of constitutive model and determination of parameters.

\section{Constitutive model}

Rubber material is super elastic material, and the model which reflects stress-strain relationship is called constitutive model. Constitutive problem is basis of study mechanical properties of materials. In the present study, the constitutive model of rubber materials is usually determined by the method of experiment. According to sufficient experimental data, determined constitutive relation of material, and there are usually two methods, that are, the statistical thermodynamics method and the continuum mechanics method based on phenomenological theory.

Based on thermodynamic statistics method common constitutive models are Arruda-Boyce model and Vander Waals model. The practice proved that it has large limitation. Based on continuum mechanics method considered that mechanical prope- rties of rubber materials are isotropic when no deformation occurs, and use strain energy function $\mathrm{W}$ of the unit volume to described basic properties of rubber materials. Strain energy function $W$ is usually expressed as function of principal elongation ratio $\lambda_{i}$ or three strain invariants of the deformation tensor $I_{i}$.

For decades of rubber constitutive research, research workers have proposed numerous constitutive models. Rivlin and Saunders ${ }^{[36]}$ according to systematic test research of vulcanized 
rubber, in order to ensure strain energy function in uniaxial, biaxial and shear test have good consistency, proposed their own model. Blatz $\mathrm{P} \mathrm{J}^{[37]}$ to overcome the phenomenon of volume locking in the nonlinear numerical simulation of large deformation of rubber material and non compressibility hypothesis of rubber material caused constitutive constraint, discussed the method of establishing constitu- tive relation of compressible rubber material, presented the constitutive relation for almost incompressible rubber materials. Tschoegl $\mathrm{N} \mathrm{W}^{[38]}$ considered that the MooneyRivlin model with higher order can better adapt to the filled and non-filled rubber materials. Experimental verification, Mooney-Rivlin model in a certain strain range correlations are very good, and than Neo-Hookean model more in line with actual behavior of rubber, but can't describe large deformation material hardening behavior ${ }^{[39]}$. Ogden $\mathrm{R} \mathrm{W}{ }^{[40]}$ to overcome used deformation tensor invariants caused complex relationship, and presented by principal elongation characterized strain energy functi- on. Wang et al. used damage theory of Lemaitre, established fatigue damage model of rubber elastic materials ${ }^{[41]}$. Horgan ${ }^{[42]}$ proposed model use principal elongation to directly characterize strain energy function of ultimate elongation of rubber materials, can be used fit strain energy of rubber material in large strain hardening.

In the process of continuous experiment verification, summarization and applicat- ion, now research constitutive model are applied more mainly:

1)Gauss statistical model: Strain energy function

$$
W=\frac{1}{2} n k T\left(\lambda_{1}^{2}+\lambda_{2}^{2}+\lambda_{3}^{2}-3\right)
$$

Among them, $\mathrm{n}$ is the average unit volume of network number, $\mathrm{T}$ is the absolute temperature, $\mathrm{k}$ is constant.

Gauss statistical model is based on the assumption that end distance is far less than the full elongation of molecular chain, and can not be used to describe large deforma- tion and elongation process of molecular chain, and only can approximate prediction the situation of small deformation. 2)Yeoh model ${ }^{[43]}$ : Strain energy function

$$
\mathrm{W}=C_{10}\left(I_{1}-3\right)+C_{20}\left(I_{1}-3\right)^{2}+C_{30}\left(I_{1}-3\right)^{3}
$$

In style, $C_{10}, C_{20}, C_{30}$ as material constitutive constants;

Yeoh model describing the deformation range is wide, can be suitable for rubber materials with different strain state, simulation of large deformation with high precisi- on, also can better predict multiaxial experimental data, but it can't fit well with equal biaxial tensile test.

3) Neo-Hookean model: Strain energy function

$$
W=C_{10}\left(I_{1}-3\right)+\frac{1}{D_{1}}(J-1)^{2}
$$

$\mathrm{J}$ is the volume ratio of after deformation and deformation before.

Neo-Hookean model is the simplest in super elasticity model, the characteristics of material can be well simulated in small strain and medium strain, and its accuracy is almost the same as Mooney-Rivlin model.

4) Mooney-Rivlin model: Strain energy function

$$
W=C_{10}\left(I_{1}-3\right)+C_{01}\left(I_{2}-3\right)
$$

The Mooney-Rivlin model is relatively simple super elastic model, and this model is often used when precise parameters of material are unknown. The mechanical properties of materials can be well simulated when model in small to medium strain, but it can't be well described for large 
deformation and steep rise of material.

5) Ogden model: Strain energy function

$$
W=\sum_{n} \frac{u_{n}}{\alpha_{n}}\left(\lambda_{1}^{\alpha_{n}}+\lambda_{2}^{\alpha_{n}}+\lambda_{3}^{\alpha_{n}}-3\right)
$$

In style, $u_{n}, \lambda_{n}$ as material constitutive constants;

In simple tensile test, Ogden model can fit experimental data well when in large strain. It also suitable for very few shear modulus of materials and minor compression materials.

Visible, each of these constitutive models has its own advantages and disadvantages, and this limitation truly reflects the complexity or essence of rubber material characte- ristics.

\section{Selection constitutive model and parameters determination}

In recent years, constitutive models of rubber material in mature stage. Most of the work of scholars reflected for existing constitutive model through a lot of tension, compression and shear experiments modified, or for concrete rubber material determi- nation of constitutive parameters.

Liu Meng et al. ${ }^{[44]}$ applied Marc nonlinear finite element analysis program, used engineering test method, tested uniaxial tensile mechanical behavior of rubber materials, and by fitting processing obtained material constants of Mooney-Rivlin model. Wang Hong ${ }^{[45]}$ through comparison axial tension and simple shear tests with measured curves, established a new rubber material hardening model. Wang Wentao ${ }^{[46]}$ studied destruction properties of rubber isolator, designed a dumbbell type rubber specimen for tensile test, studied mechanical properties by analysis of experimental data. $\mathrm{Yu}$ Guijie et $\mathrm{al}^{[47]}$ through use filler filled with rubber materials for uniaxial tensile test, fitting obtain Mooney-Rivlin model and Yeoh model material parameters, combined with experimental data determined strain energy function model of filled rubber material. Li Hao et al. ${ }^{[48]}$ based on direct method of spline interpolation, constructed exact conformity to uniaxial and equal biaxial tensile data and large deformation of shear data super elastic potential energy, deduced the stress strain relationship under normal deformation, predicted non biaxial tensile experiment and compared with the Rivlin non biaxial tensile experimental data. Guo Hui et al ${ }^{[49]}$ by using strain energy function of incompressible rubber materials, by introducing porosity of foam material, constitutive equation of foam rubber was deduced. Based on experimental results of uniaxial compression of foamed rubber, determined the parameters of constitutive model.

$\mathrm{Hu}$ Xiaoling et al ${ }^{[50]}$ used carbon black filled rubber materials for uniaxial tension, flat stretch and biaxial tension basic deformation experiments, investigate to experim- ental data fitting ability of common super elasticity constitutive model, and discussed the super elasticity constitutive model predictive ability and model selection strategy in three basic experiments are not complete. Gong $\mathrm{Kejia}^{[51]}$ developed a based on image analysis of complete rubber material parameters testing system, and applied the system of truck tire rubber carried out uniaxial tension, biaxial tension and pure shear test. The results show that biaxial tension and pure shear test are essential for determining reliable rubber constitutive. Wang Zhiyu ${ }^{[52]}$ according to rubber specimen in uniaxial tensile tests obtained tension and deformation value experimental data, use of multiple regression method obtained rubber material Mooney-Rivlin model three type model constants.

In order to study the effect of carbon black on mechanical behavior of rubber materials, Li Qing et $\mathrm{al}^{[53]}$ for 9 kinds of different volume fraction of carbon black filled rubber material carry out quasi static uniaxial tensile test, and according to test data fitted Mooney rubber constitutive constants by using the least squares method. Wang Wei et al. ${ }^{[54]}$ use under uniaxial tension, biaxial tension and 
planar shear rubber material test data of four kinds of different combination methods were mainly used describe rubber super elastic Yeoh model and Ogden third-order model fitting, and draw material parameters of the two models. Zhang Ping ${ }^{[55]}$ for rubber specimen carried out uniaxial tension, biaxial tension and planar tension test, obtained stress- strain curves of the specimens under various stress states, application the method of least squares fitting obtained different rubber super elastic constitutive model parame- ters.

The mechanical behavior of rubber materials is complex and the constitutive relation is non-linear, an accurate description of its mechanical properties is difficult. Accurate characterization of rubber material properties depend only on including tension, compression, shear and volume test basic test, is not enough ${ }^{[56]}$.

\section{Conclusions}

(1) The uniaxial load experiment is easy to realize, and it has accumulated plenty of experience, data and conclusions and criteria for both quasi-static and dynamic loads, however, the actual engineering rubber materials is in a complicated stress state, simplify to simple stress state or the conclusion and criterion of uniaxial load are extended to multiaxial load, there will inevitably be a variety of deviations.

(2) The multiaxial load is applied to fatigue study of rubber materials, but the methods of realizing the multiaxial load is still not much.

(3)Rely on uniaxial tension test, compression test, shear test, biaxial tension experim- ent, and planar tensile test established the constitutive model of rubber material each has its limitation. Different experiments adopt different sizes and styles specimens, don't consider the size effect and surface effect, and then integrate the data, lack of convincing.

(4) Due to the complexity of multiaxial loading, experimental study of quasi-static multiaxial loading is seldom reported, and experimental study of equivalent concept of multiple stress state is also lack of.

\section{References}

[1] Cadwell S M, Merrill R A,Sloman C M,et al. Dynamic fatigue life of rubber [J]. Rub- ber Chem \&Tech, 1940,13(5): 304-315.

[2] Fielding J.H. Flex life and crystallization of synthetic rubber [J]. Ind Eng Chem, 1943, (35): 1259-1261.

[3]Beatty J. R. Fatigue of rubber[J]. Rubber Chemistry and Technology.1964,37:1341

[4] Lee B.L, Ku B.H, Liu D.S, et al. Fatigue of cord-rubber composites (II): Strain- based fatigue failure criteria, Rubber Chemistry and Technology,1998, 71(2):866-888.

[5] Abraham F,Alshuth T,Jerranms S.The effect of minimum stress and stress amplitude on the fatigue life of non strain crystallizing elastomers[J]. Mat\&Des, 2005,26(3): 239-245.

[6] Mars W.V, Fatemi.A. Fatigue crack nucleation and growth in filled natural rubber. Fatigue Fract Engng Mater Struct.2003,26:779-789.

[7] Ellul M. D, Mechanical fatigue. In: A. Gent, Editor, Engineering with rubber, How to design rubber components, Carl Hanser Verlag, Munich, 1992.

[8] Y.Y. Liu, Z.H. Tian, Z.M. Wan, et al. Fatigue damage characteristics of rubber composites under cyclic loading [J].Rubber industry,2003,50(12):713-715.

[9] Lee M. P, Moet A. Analysis of Fatigue Crack Propagation in NR/BR rubber blend [J]. Rubber 
Chemistry and Technology, 1993, 66:304-316.

[10] Hocine N.A, Abdelaziz M.N, Mesmacque G. Experimental and numerical invest- igation on single specimen methods of determination of $\mathrm{J}$ in rubber materials[J].Inter- national Journal of Fracture.1998, 94:321-338

[11] MARS W V. Multiaxial fatigue of rubber [D]. Toledo: The University of Toledo, 2001. [12] Roberts B J, Benzies J B. Relationship between uniaxial and equibiaxial fatigue fatigue ingum and carbon-black-filled vulcanizates[J]. Plastics and Rnbber: Materials and Applications, 1978,3(49-54).

[13] Ro H.S. Modeling and interpretation of fatigue failure initiation in rubber related to pneumatic tires [D]. Purdue University, 1989.

[14] Yamashita S. Selecting damping materials (service environment, strain and endurance) [J]. International Polymer Science and Technology.1992, 19(4):41-56.

[15] Zine A., Benseddiq N., Abdelaziz M. NaIT, et al. Prediction of rubber fatigue life under multiaxial loading[J]. Fatigue\&Fracture of Engineering Materials \& Structures, 2006,29(3): 267 -278 .

[16] Zine A., Benseddiq N., Nait Abdelaziz M. Rubber fatigue life under multiaxial loading: Numerical and experimental investigations[J]. International Journal of Fati- gue, 2011,33(10): 1360-1368.

[17] Kim W. D., a H. J. Lee, a J. Y. Kim, et al. Fatigue life estimation of an engine rubber mount [J]. International Journal of Fatigue, 2004,26 (553-560).

[18] Chang-Su Woo, Wan-Doo Kim, Jae-Do Kwon. A study on the material properti- es and fatigue life prediction of natural rubber component[J]. Materials Science and Engineering: A, 2006, 483-484 (376-381).

[19] W. Zhang, C.J. Song, H.B. Zhang, et al. Experimental study on mechanical prop- erties of vulcanized rubber materials [J].weapon materials science and Engineering, 2010, 33(6):17-18. [20] S.X. Yin, S.W. Xiao, Y.J. Huang, et al. Influence of pre compression on the fatigue life of rubber ball joints [J]. Mechanical strength, 2012,34(3):384-388

[21]S.H. Xiao, W.T. Wang, J.L Huang, et al. Fatigue life prediction of rubber isolator under uniaxial tension state [J].vibration and impact,2014,33(5):24-31.

[22] X.L. Wang, W.B. Shangguan,et al. Filled rubber material uniaxial tensile fatigue test and fatigue life model [J]. Mechanical Engineering Journal, 2013, 49(14): 65-73.

[23] Mars W V, Fatemi A, Multiaxial fatigue of rubber: Part II: experimental observa- tions and life predictions, Fatigue Fract Eng Mater Struct 2005, 28:523-538.

[24] Saintier N, Calletaud G, Piques R, Multiaxial fatigue life prediction for a natural rubber, International journal of fatigue, 2006, 28:530-539.

[25] Li C G, Steif P S.Multiaxial cyclic response of filled rubber[J].Rubber Chemistry and Technology, 2000 , 73 :193-204.

[26] Mars W V, Fatemi A.Observations of the constitutive response and characterizat- ion of filled natural rubber under monotonic and cyclic multiaxial stress states[J]. ASME J .Eng. Mater. Technol, 2004,126:19-28.

[27] Saintier N., Cailletaud G., Piques R. Crack initiation and propagation under multiaxial in a natural rubber[J]. International Journal of Fatigue,2006,28(1): 61-72.

[28] Andriyana A., Saintier N., Verron E. Configurational Mechanics and Critical Plane Approach: Concept and application to fatigue failure analysis of rubber like materials [J]. International Journal of Fatigue,2010,32(10): 1627-1638.

[29] Andriyana A., Verron E. Prediction of fatigue life improvement in natural rubber using 
configurational stress[J]. International Journal of Solids and Structures, 2007, 44(7-8): 2079-2092 [30] W.W. Yu, X. Chen, L. Yan. Mechanical behaviour of rubber under multiaxial cyclic loading[ J] .China Rubber Industry ,2007, 54(8):500-503.

[31] Z.P. Ding, R.H.a Yang,Y.J. Huang. Prediction of fatigue crack growth life of rubber ball hinge [J]. Journal of Hunan University of Technology, 2013, 27(2): 48-53.

[32] Q. Li, J.C. Zhao, B. Zhao. Fatigue life prediction of a rubber mount based on test of material properties and finite element analysis[J]. Engineering Failure Analysis, 2009, 16(7), 2304-2310.

[33]Y.P. Wang. The fatigue life and microstructure of rubber materials[D]:Tianjin university, 2007.

[34]W.T. Wang, W.B. Shangguan. Super elastic constitutive model of rubber vibration isolators static characteristics[J].Automotive engineering prediction,2012,(6):544-550.

[35] W.T. Wang, W.B. Shangguan, et al. Based on linear fatigue cumulative damage rubber mounting fatigue life prediction [J]. Chinese Journal of Mechanical Engineer- ing, 2012,(10):56-65. [36] Rivlin R S, Saunders D W. The free energy of deformation for vulcanized rubber. Ph i.l Trans. Roy. Soc. 1951,243-251.

[37] Blatz P J, Ko W L. Application of finite elastic theory to the deformation of rubber materials [J]. Transact ion of the Society of Rh eology, 1962, 6( 2): 223-251.

[38] Tschoegl N W. Con stitutive equations for elastomers [J].Journal of Polymer Science, 1971

(12) : 1959-1970.

[39]X.F. Li, X.X. Yang. The constitutive model of super elastic for rubber materials [J]. Elastomer,2005,15(1):50-58.

[40] Ogden R W. Large deformation is otropic elasticity: On the correlation of theory and experiment $\mathrm{f}$ or incompress iblerubber-like solids [A] . Proceedin g of the Royal Society of London[ C]. London, UK: 1972. 565- 584.

[41] B. Wang, H.B. Lu, Gyu-ho Kim. A damage model for fatigue life of elastomeric materials [J]. Mechanics of Materials. 2002, 34: 475-483.

[42] Horgan C O, Saccomandi G. C on stitutive modeling of rubber-like and biological material with limiting chain extensibility[J] . Mathematics and Mechanics of Solids, 2002,7:353-371.

[43] Arruda Boyce.Comstitutive model of rubberelasticity:a review[J]. Rubber Chemis- try and Technology, 2000,73:504-523.

[44] M. Liu, Q.C. Wang, G.Q. Wang. Determination of material constants constants in rubber Mooney-Rivlin model [J]. rubber industry,2011,58(4):241-245.

[45] H. Wang, Y.F Zhu, Constitutive model and finite element analysis of rubber mat- erial hardening [J]. Journal of Wuhan Institute of Technology, 2008, 30(1): 34-36.

[46] S.H Xiao, W.T. Wang, J.L. Huang. Fatigue life prediction of rubber isolator under uniaxial tension state [J]. vibration and impact, 2014, 33(5): 24-30.

[47] G.J Yu, M Gao, X.C. Wei. Study on the mechanical properties of natural vulcani- zed rubber filled with the fillers filled with super elastic rubber tube [J]. lubrication and seal, 2013, 38(10):

25-29.

[48] H. Li, Y.Y. Zhang, H. Xiao. Fitting the rubber material non equibiaxial deformat- ion explicit data super elastic potential [J]. Applied Mathematics and mechanics, 2013, 34(5): 470 -479.

[49] H. Guo, W.J. Hu, J.L Tao. The super elasticity constitutive model of foam rubber materials [J]. Journal of mechanics,2013,30(4):575-579.

[50] X.L. Hu, X. Liu, M. Li. Selection of the selection strategy for the constitutive model of carbon black filled rubber [J]. engineering mechanics, 2014, 31(5):34-42.

[51] K. Gong, Y.T. Wei, J.X. Ye. Filled rubber hyperelastic constitutive parameter test and application of [J]. Engineering Mechanics,2009,26(6):193-198. 
[52] Z.Y. Wang, A.W. Wang. Multivariate linear regression method to determine the rubber Mooney-Rivlin model constant[J].Journal of Naval Engineering University, 2011,23(2)18-21.

[53] Q. Li, X.X. Yang. Macro meso mechanical behavior of carbon black filled rubber composites [J].Journal of Mechanical Engineering,2013,49(18):132-139.

[54]W. Wang, S.Yan. Determination of material parameters in rubber super elasticity constitutive model [J]. rubber industry,2014,61(8):453-457.

[55] P. Zhang, G.Z. Chai, X.Y. Pan. Study on the static characteristics of rubber isola- tor [J].Vibration, testing and diagnosis, 2010, 30(2): 105-110.

[56] Miller K.Testing elastomers for hyperelastic mateial models in finite element analysis [M]. Rubber Technology International. UK: UK \& International Press, 1999. 